\title{
A parametric-assisted method for 3D generation of as-built BIM models for the built heritage
}

\author{
M. Zheliazkova, R. Naboni \& I. Paoletti \\ $A C T L A B$, Department ABC, Politecnico di Milano, Italy
}

\begin{abstract}
The paper outlines a parametric-assisted method for the 3D reconstruction and creation of BIM models for the built heritage. The research implements the emerging paradigms of open sourcing, cloud computing and interoperability, employing low-cost technologies (digital photogrammetry) and open source software (Grasshopper for Rhinoceros) which can ease the accessibility to a potential reuse of heritage, typically requiring high specialists and expensive equipment. The research examines the abandoned Albergo Diurno "Venezia" in Milan, heritage with a unique architectural value - a blend of Liberty and Art Deco styles. The process of 3D reconstruction of the ceiling is described. Custom algorithms have been developed to automatically rebuild the complex and irregular geometry from mesh, towards the creation of a NURBS-based 3D model. It is shown how the proposed methodology can streamline the process of data elaboration by reducing arbitrary operations and improve accuracy to preserve geometric irregularities. The associative model allows the automatic improvement in the model definition when more precise input data is feeding the algorithm, offering the opportunity to relate the precision of BIM models in accordance with the needed level of detail (LOD).

Keywords: photogrammetry, point cloud, automated $3 D$ reconstruction, as-built BIM, algorithmic process, interoperability.
\end{abstract}

\section{Introduction}

This paper is part of a research agenda aiming at establishing links between the currently disconnected domains of cultural heritage, adaptive reuse and the use of innovative technologies, placed under a common BIM platform. Nowadays, the 
design and construction sector is moving towards a full software interoperability, favouring the exchange of shared knowledge in design and manufacturing. Advanced design, fabrication tools and free/open source software (F/OSS) could generate important benefits in the realm of cultural heritage. Their implementation can drive the shift from strictly procedural preservation programs towards the development of bespoke building processes and solutions able to address specific problems in the reuse of built heritage. The approach presented here focuses on digital photogrammetry and open source software for an accurate data elaboration to facilitate the access to the cultural heritage, replacing the need of specialists and high-end equipment (laser scanners, total stations, specific software for processing large point clouds). The broader goal of this research is to produce a highly accurate Building Information Model (BIM) as a multi-use platform.

The relevance of BIM as a digital representation of physical and functional characteristics of a patrimony has already been introduced and widely discussed (Arayici [1]). BIM is recognized as an important technology addressing the growing demand for a multi-disciplinary knowledge base, essential to the documentation and management of a building's life-cycle processes (Fai et al. [2]). Our research intends BIM as a collection of many data-rich models and databases, which can be easily and effectively shared between disciplines and their platforms and software programs (Kensek [3]). Within this definition, an "asbuilt" BIM model, as the state of the building at the moment of the survey, integrates the initial survey and geometry of a heritage, the results of analyses performed prior to the interventions, and the design and all fabrication documents of the reuse. Finally, the BIM model(s) serve for the future facility management, monitoring and repair.

Despite the fast advancements in the development of the BIM technology, current software are still unable to handle complex and irregular geometries. For this reason most approaches for reconstruction and reverse engineering use NURBS modelling tools. However, the existing methods are time-consuming and involve several plugins and advanced modelling skills. In this sense, the associative 3D reconstruction methods later described allow for the creation of models with differential resolutions and accuracy, which can be produced in different stages. This opens new opportunities in understanding the survey and 3D reconstruction as a flexible and upgradable process, according to multidisciplinary needs.

\section{An enhanced workflow for creating an "as-built" BIM}

The process of creating an "as-built" BIM usually involves three phases. The first is the survey and data collection, followed by a second phase of data processing, object recognition and semantic labelling - the most intensive part involving manual modelling. The last phase is the compiling of the final semantically enriched 3D model (Volk et al. [4]). The methodology proposed in this paper aims at enhancing the current workflow for creating an "as-built" BIM introducing algorithmic modelling tools and open source software in the phase of data 
processing, considered fundamental for the precision of the final model. In Grasshopper custom algorithms are developed to automatically rebuild complex shapes and irregular geometries from polygonal mesh, towards the creation of a NURBS-based 3D model. The latter is then loosely converted into BIM objects through already consolidated procedures where further layers of information are added (Oreni et al. [5]).

The methodology outlined in this paper relies on digital photogrammetry for the virtual representation. The use of image-based 3D reconstruction software generates point cloud densities comparable to the LiDAR ones (Rinaudo et al. [6]). Common BIM software is still unable to manage the huge quantities of data coming from laser scans or photogrammetric surveys, which makes inevitable the use of external intermediate software tools converting datasets in high-defined polygonal meshes (Hichri et al. [7]). The incapability of most BIM software to reconstruct free-form shapes or to register minor irregularities in the building elements poses a great challenge when dealing with heritage. Our approach adopts NURBS (Non-uniform Rational Basis Spline) as the most appropriate mathematical model for representing the complexity present in historic buildings. NURBS uses several industry-standard methods for exchange, which favours the easier transfer of data among different software. The model developed in Grasshopper is associative i.e. allowing the automatic improvement in the definition when more precise input is feeding the algorithm, offering the opportunity to relate the precision of BIM models in accordance with the needed LOD. Moreover, Grasshopper is a free tool that can process automatically great amounts of data, reducing arbitrary and manual operations and the possibilities for mistakes.

The methodology has been tested with the case study of Albergo Diurno "Venezia" in Milano. The focus was the reconstruction of the ceiling of the entrance hall comprised of twenty-one sail domes connected by cylindrical vaults.

\section{Data acquisition case study: Albergo Diurno "Venezia"}

\subsection{Data capturing using digital photogrammetry}

The close range survey approach has been profoundly renovated with the development of LiDAR and multi-image matching techniques allowing the designer to work with datasets of millions of points. After analysing the most common data capturing techniques (laser scanning, photogrammetry, RFID and barcode tagging), digital photogrammetry was chosen as the fastest, most costoptimized and accessible method for acquisition. Moreover, it has been proved photogrammetry can provide useful result even without LiDAR integration (Rinaudo et al. [6]). The opposite case is less probable, as the LiDAR technology uses not self-controlled strategy, i.e. the three coordinates of a generic point are estimated by using just three independent measurements. Photogrammetry estimates the coordinates by using at least four independent measurements. 


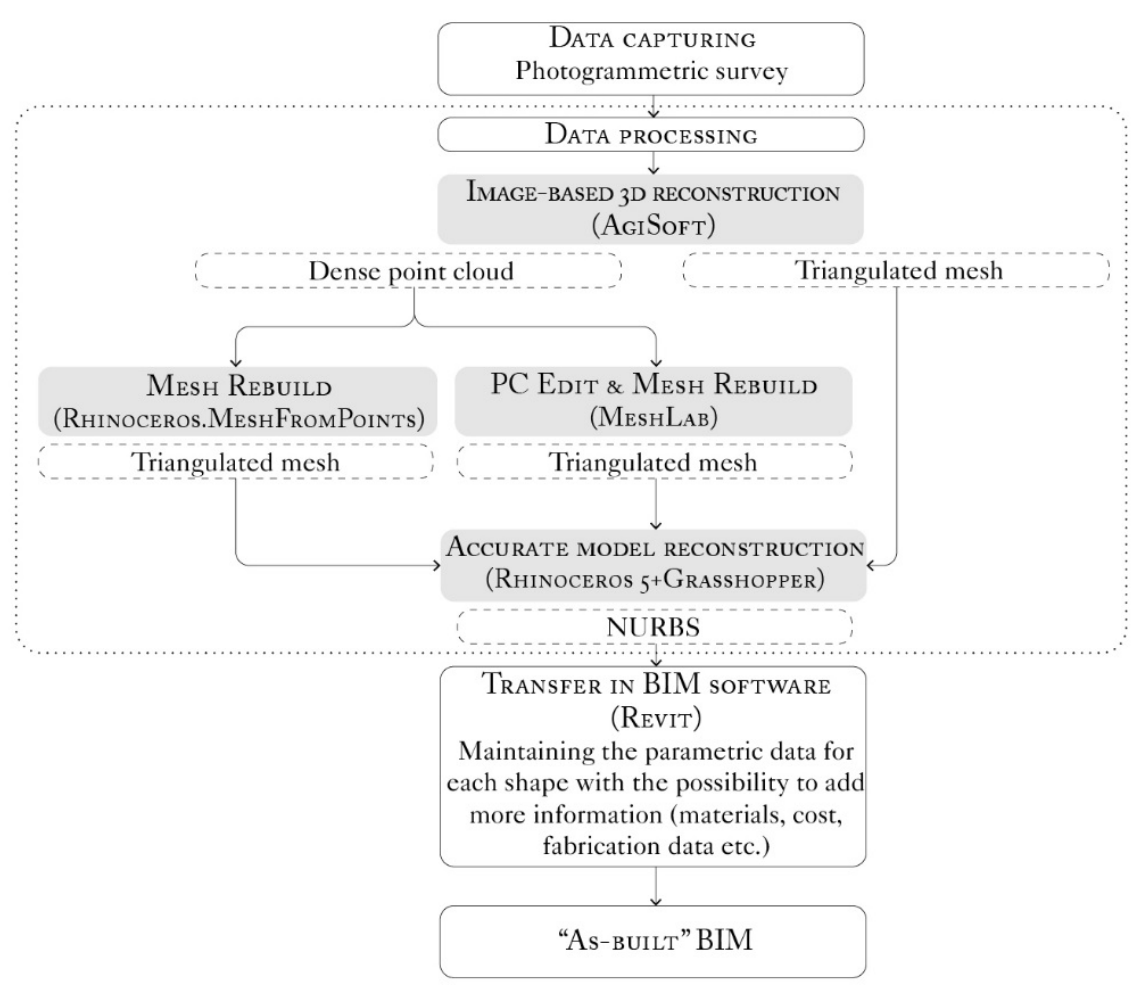

Figure 1: Upgraded workflow for creating an as-built BIM model.

\subsection{Case study: Albergo Diurno "Venezia"}

Albergo Diurno "Venezia" is an iconic building located under Piazza Oberdan, in the city centre of Milan, Italy built in 1925 by Eng. Marcello Troiani. It has been of interest to the research as one of the most striking examples of blending Liberty and Art Deco in Italy. Diurno was a hosting a curious combination of commercial activities like ticket selling, bank, post office, and others with public baths used for refreshment by the travelling workers. This nowadays obsolete function, as well as its unique architectural characteristics, require a highly specific reuse project. The proposal by the local municipality is to be reused as a museum of the Italian cinema. A challenging transformation which became an opportunity for developing a new procedure which could be directly implemented. The presented methodology focuses on the entrance lobby and the atrium hall, which feature the most important Art Deco decoration and are more spatially and geometrically articulated than the rest of the building. 


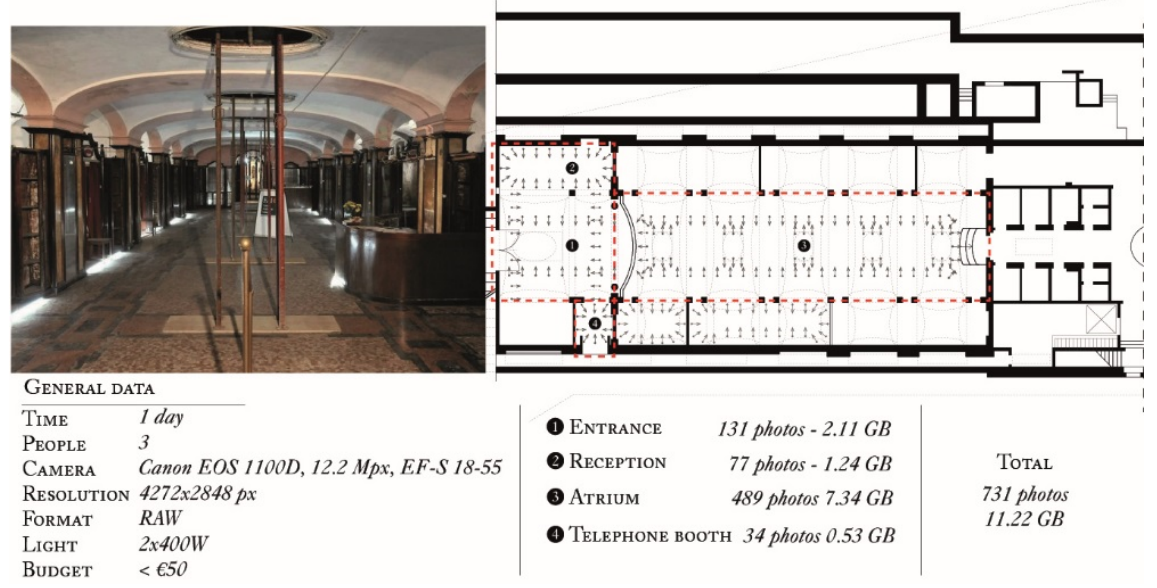

Figure 2: Albergo Diurno "Venezia" survey and general information.

\subsection{Photogrammetric survey}

The photogrammetric survey of Albergo Diurno had been challenging mainly due to the fact the building is located underground and no electric current was provided. However, the images taken with the use of portable artificial lighting were defined enough to obtain an accurate dense point cloud. Photogrammetry proved to be a reliable method for surveying cultural heritage even in unfavorable conditions.

The data acquisition has been carried out during a single one-day-campaign by a team of three people. Two standard projectors of $400 \mathrm{~W}$ were used. The camera - Canon EOS 1100D - has a $22.2 \times 14.7 \mathrm{~mm}^{2}$ complementary metal-oxide semiconductor (CMOS) sensor divided into $4272 \times 2848$ or 12.2 mega pixels, with each pixel having $5.2 \mu \mathrm{m}$ pixel size in both dimensions. The photos were taken with $60 \%$ horizontal and $80 \%$ vertical overlap, ensuring each part of the building interior is present on at least two different photos.

\section{A parametric-assisted method for 3D reconstruction}

\subsection{Data elaboration}

\subsubsection{Image-based reconstruction}

For the elaboration of the survey data was used an image-based modelling software. The multi-view 3D reconstruction technology operates on arbitrary images and is efficient in both controlled and uncontrolled illumination conditions. Advanced surface measurement algorithms can get similar results to range sensors, but in a cheaper, faster and simpler way (Remondino [8]).

After comparing various commercial and F/OS image-based reconstruction software, the results proved AgiSoft PhotoScan as the most effective and fast solution for creating a dense point cloud, table 1 . The software automatically 
processes the uploaded photos without any input of camera parameters or manual photo alignment, in difference with other 3D photogrammetric tools. The output is a highly accurate point cloud (up to $1 \mathrm{~mm}$ tolerance) from which a polygonal textured 3D model was automatically generated.

Table 1: Analysis of commercial capturing, processing and modeling software.

\begin{tabular}{|c|c|c|c|c|c|}
\hline $\begin{array}{l}\text { Commercial } \\
\text { Software }\end{array}$ & $\begin{array}{l}\text { LASER } \\
\text { SCANNING }\end{array}$ & $\begin{array}{c}\text { Photo } \\
\text { GRAMMETRY }\end{array}$ & $\underset{\text { INTEGRATION }}{\text { BIM }}$ & $\begin{array}{c}\text { CAD } \\
\text { INTEGRATION }\end{array}$ & Notes \\
\hline Australis & - & $\mathrm{X}$ & No & Yes & Export to DXF and ASCII \\
\hline Canoma & - & $\mathrm{X}$ & No & Yes & Surface texturing to CAD objects \\
\hline INOVxRealityLinx Mode & lel - & $\mathrm{X}$ & No & Yes & Plant application \\
\hline NuBAU freac & $\mathrm{X}$ & $\mathrm{X}$ & Yes & No & Interactive creation of floorplans \\
\hline ; & $\cdots$ & $\cdots$ & $\cdots$ & $\cdots$ & -- \\
\hline Autodesk Image Modeler & - & $\mathrm{X}$ & Yes & Yes & Architectural application; DWG file export \\
\hline PhotoModeler & - & $\mathrm{X}$ & Yes & Yes & Export in DXF, textured surfaces and elevations \\
\hline Menci & - & $\mathrm{X}$ & Yes & Yes & Architectural application; point cloud \\
\hline AgiSoft & - & $\mathrm{X}$ & Yes & Yes & Architectural application; point cloud \\
\hline Autodesk ReCap. & - & $\mathrm{X}$ & Yes & Yes & Cloud based; point cloud \\
\hline $123 \mathrm{D}$ Catch & - & $\mathrm{X}$ & Yes & Yes & \\
\hline Insights $3 \mathrm{D}$ & - & $\mathrm{X}$ & Yes & Yes & Free; open source; for small object or sculptures \\
\hline VisualSFM & - & $\mathrm{X}$ & Yes & Yes & Free; open source; mainly facades \\
\hline
\end{tabular}

4.1.1.1 Dense point cloud generation The dense point cloud has been obtained following the linear workflow in AgiSoft. The software first processes the images, aligning them and matching common points leading to a sparse point cloud and then builds a dense point cloud based on the estimated camera positions and the pictures.

For the generation of the model of the vaulted ceiling of Albergo Diurno, 811 images converted from RAW to TIFF were processed to a dense point cloud of 8 659402 points - with a resolution $0.00394712 \mathrm{~m} / \mathrm{pix}$ and point density of 64185.9 $\mathrm{pts} / \mathrm{m}^{2}$. The software allows full control of the user over the precision which were set to "high", i.e. applying image size reduction by factor of 4 . The estimated reprojection error is $16.2597 \mathrm{px}$.

4.1.1.2 Polygonal 3D model reconstruction The conversion of point clouds to polygonal models is essential for the 3D restitution because of the very accurate results it gives, mainly because the meshes are generated through direct interpolation of the points in the dataset (Berger et al. [9]). The common procedures for $3 \mathrm{D}$ model reconstruction are using surface and volumetric representation. For the surface representation a B-Rep describes shapes by a set of surface vectors extracted from the point cloud, usually constituting the limits of surface (Baumgart [10]). Volumetric representations describe shapes with geometric solids known as CSG (Constructive Solid Geometry), which build complex shapes starting from simple geometric primitives and using Boolean operations (Lin and Chen [11]). Both of these involve additional software and plug-ins and many manual operations, making the whole process costly and time consuming. Moreover, during the manual manipulation of points a lot of the initially obtained data in the cloud is omitted. With the methodology proposed here, a mesh is created automatically from the point cloud, as the precision and the number of vertices are user-defined. The problem of the reconstruction to NURBS 
is solved with algorithms using the generated mesh as a direct input and produce a smooth NURBS surface out of it.

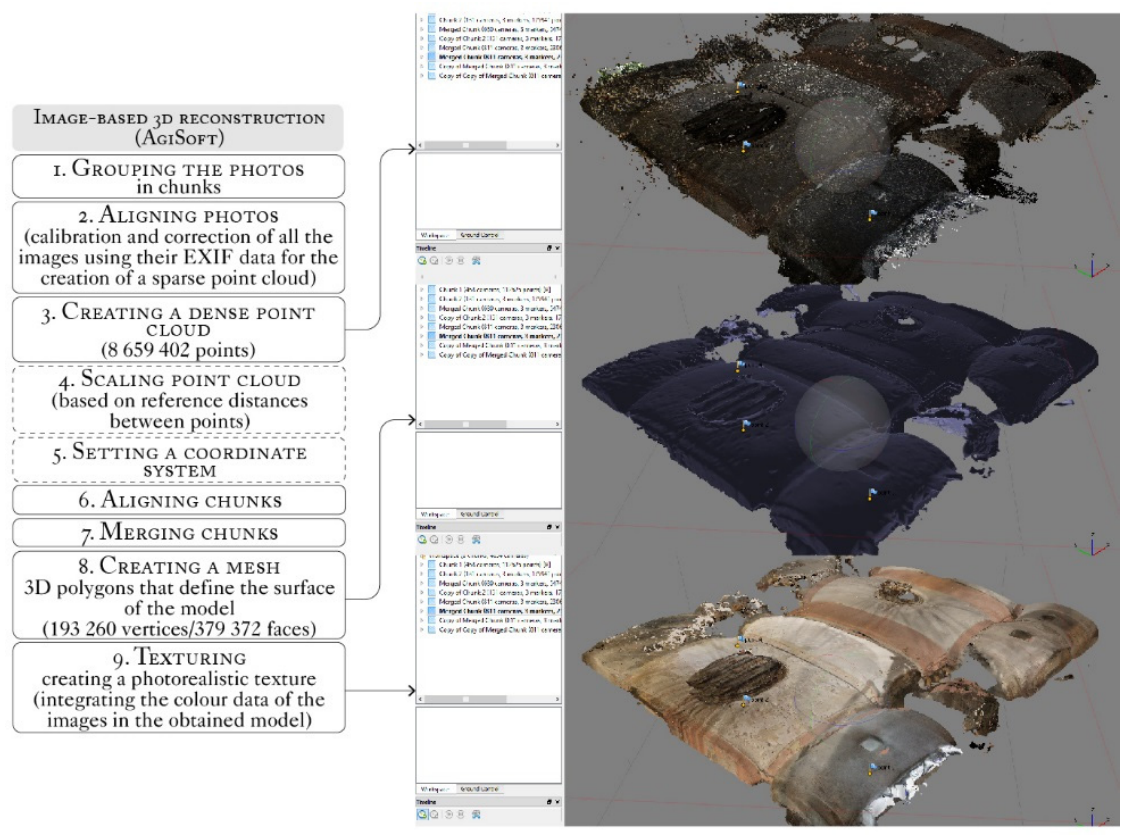

Figure 3: Workflow for obtaining the point cloud and the textured mesh in AgiSoft PhotoScan.

The polygonal model of the ceiling of Albergo Diurno has been calculated based on the number of points in the dense point cloud. The quality input was set to medium, which represents a ratio of $1 / 15$ related to the number of points in the dense point cloud.

\subsubsection{Polygonal 3D model reconstruction with F/OSS}

For achieving a streamlined workflow, custom algorithms were developed and tested on different polygonal models, generated with different tools. The final NURBS surfaces were compared and evaluated. The goal was to define the best application for each tool, as well as to understand the potentialities of the open standards for the exchange among various platforms. Two F/OS software were tested, which use two different algorithms for mesh generation.

The first one is MeshLab, an open source, portable and extensible system for the processing and editing of unstructured triangular meshes, which also deals with automatic reconstruction from point. The tested Ball-Pivoting Algorithm (BPA) requires relatively small amount of computer memory, it is time eff cient and produces highly accurate results. The BPA follows a simple principle: three points form a triangle if a ball of a user-specif ed radius touches them without containing any other point. The ball pivots around an edge until it touches another point, 
forming another triangle (Bernardini et al. [12]). For the case study of Albergo Diurno this algorithm has been run on a dataset of 1607929 points to generate a triangular mesh of 218693 faces. The intersecting ball to construct the mesh had a diameter of $0.041419 \mathrm{~m}$.

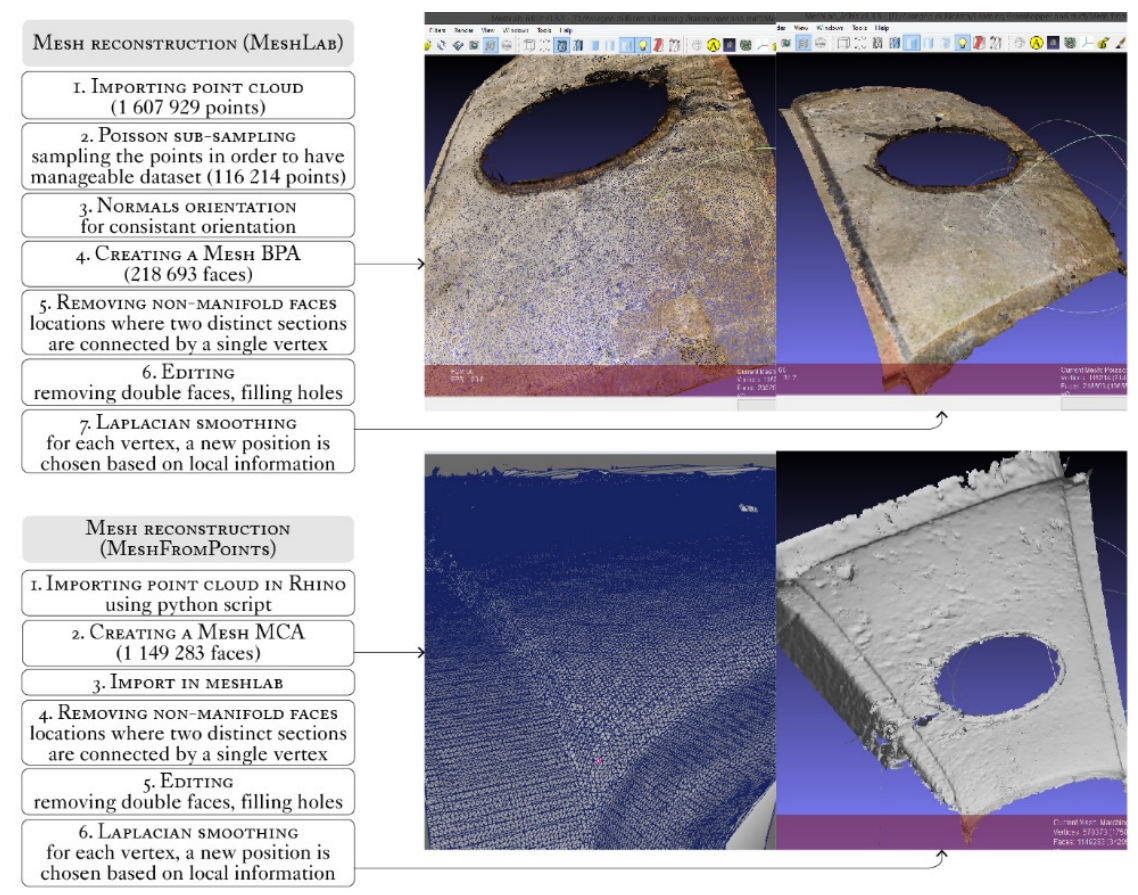

Figure 4: Workflow for obtaining a mesh with MeshLab and MeshFromPoints.

The second tool is MeshFromPoints, a free Rhinoceros plug-in using the Marching Cube (MC) algorithm which converts the point cloud into a volumetric (voxel) distance field and reconstruct the implicit surface. It proceeds through the scalar field, taking eight neighbor locations at a time (thus forming a cube), then determining the polygons needed to represent the part of the isosurface that passes through this cube. The individual polygons are then fused into the desired surface (Dietrich et al. [13]). In comparison with the previous algorithm, this method generated a mesh with much more faces (1 149 283) in less time.

However, one of the key shortcomings of this approach is the quality of the resulting mesh, which had many poorly shaped and degenerate triangles. The mesh was cleaned and edited in MeshLab.

\subsection{Custom-made algorithms for an automated 3D reconstruction from mesh}

The conversion of meshes into smooth surfaced NURBS objects is not a direct but rather complex and difficult process. There are tools which convert meshes into 
NURBS surfaces, yet as a mesh is a series of discrete facets, the algorithm creates a surface for each mesh face resulting into a polysurface hardly useable in a BIM environment. A number of specialized reverse engineering software packages, tools and Rhinoceros plugins attempt at generating NURBS without facets multiplication. Usually this software is characterized by a high range price. The methodology here described uses different algorithms for the automated reconstruction of accurate NURBS surfaces from meshes. The opportunity to create custom algorithms, versus using already developed tools, allows to interpret specific morphological characteristics of the building with the human comprehension of the construction logics and the topological characteristics.

\subsubsection{An algorithm for reconstructing NURBS surface from contour lines}

In order to test the different output coming from the three different meshes, two strategies for creating a NURBS surface have been developed.

The first algorithm was used for reconstructing a series of domes, as well as for the mesh of the first dome generated in MeshLab. It is based on extracting contour lines, which describe the geometry in both $\mathrm{X}$ and $\mathrm{Y}$ direction. The number of the contour lines is user-defined, as the more the contours, the more precise the final result (as it interpolates more data from the mesh). Working with the case study of the ceiling of Albergo Diurno comprised of multiple domes connected with vaults, it was clear that their boundaries were essential for correctly constructing the geometry. A closed B-rep, described by 4 user-defined input points intersects the mesh in the exact domes' boundaries. The B-rep is also used for cutting the contour curves which are then reordered, rebuilt and the non-fitting ones removed. The test shows the method is applicable to meshes with unfilled faces, as the algorithm interpolates in new curves the points of the initial contour, thus automatically filling the missing face, fig. 5. Finally the surface is created from the network of the rebuilt contour curves.

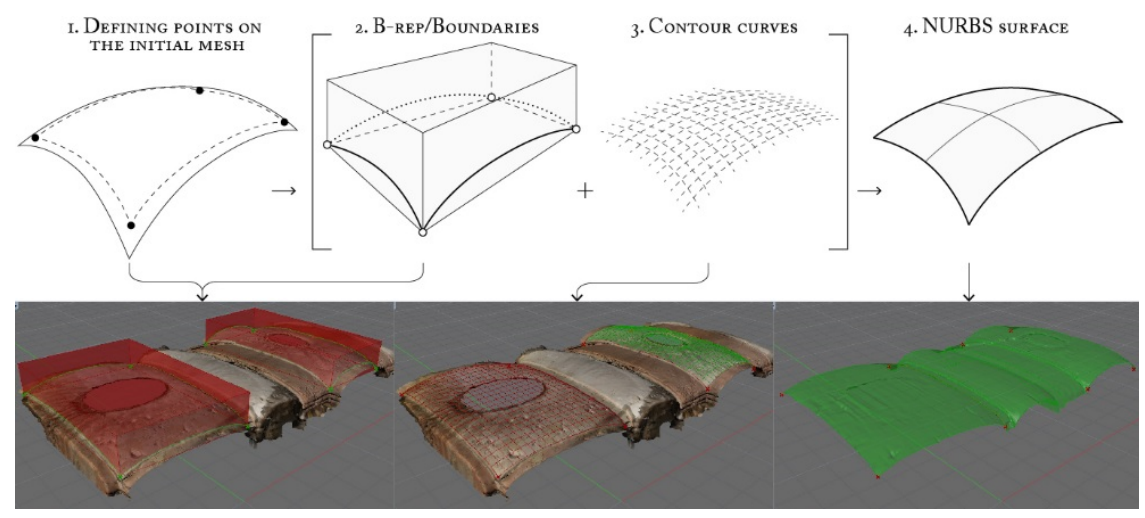

Figure 5: Parametric reconstruction from contours.

An algorithm was used to automatically create the surfaces between the domes and the vaults, connecting the two neighbouring edges of the vault and the dome 
respectively. Thus the precision of these surfaces is directly related to the precision of the surfaces of the domes and the vaults.

\subsubsection{An algorithm for reconstructing NURBS surface from grid of points}

The second strategy, applied to the mesh generated with the MC algorithm, is using points for the surface reconstruction, and avoiding the creation of contour lines. In this process, a series of points in $\mathrm{X}$ and $\mathrm{Y}$ direction and forming a regular grid, is projected directly onto the mesh. The projected points, enclosed in a B-rep defined by 4 user-input points describing the dome's boundaries, still form a regular grid but with varying $\mathrm{Z}$ value. From those points, through direct interpolation, a smooth NURBS surface is created, fig. 6. The number of the points can vary, depending on the input the user defines, as of course, the more the points, the more precise the final surface. This method, though, is applicable mainly to uninterrupted meshes without unfilled holes.

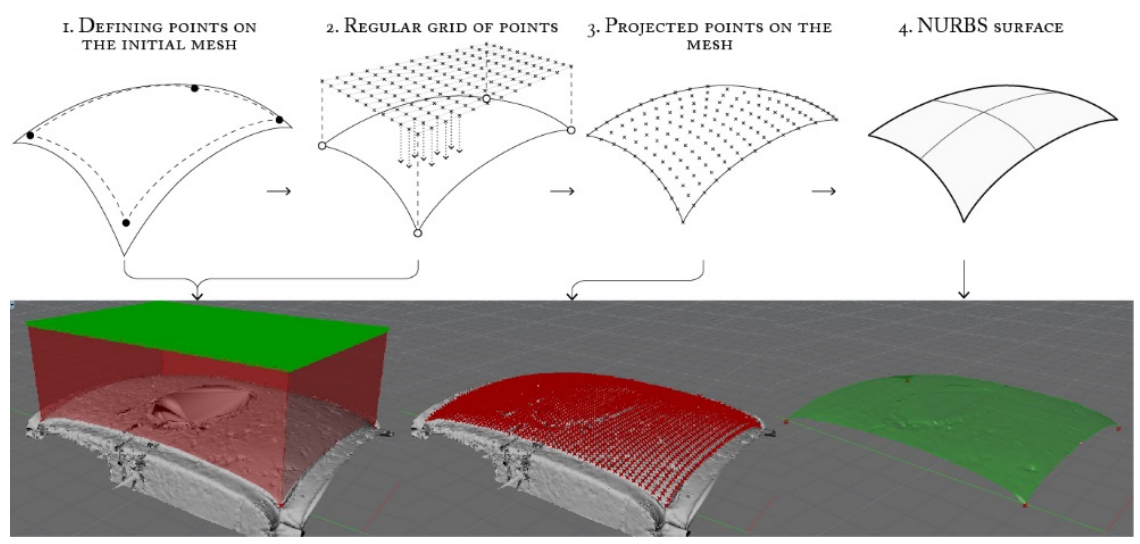

Figure 6: Parametric reconstruction using grid of points.
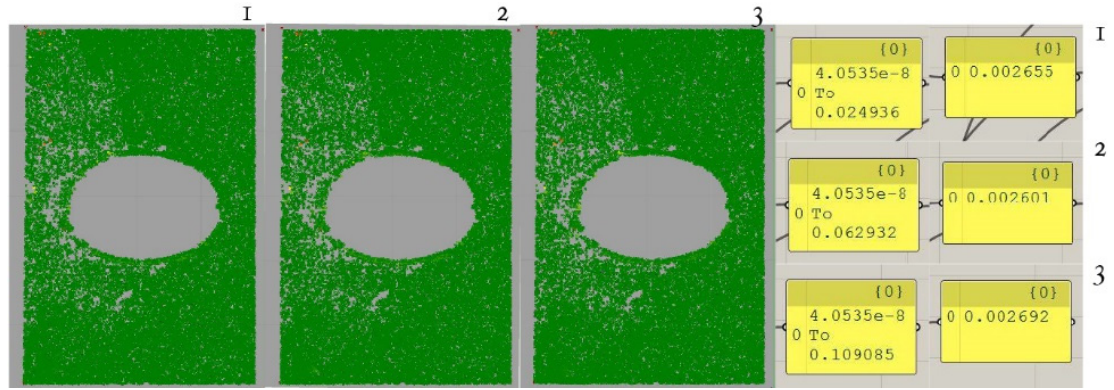

Figure 7: Points projected and average value of the distances: 1. mesh from AgiSoft; 2. BPA mesh; 3. MC mesh. 
While a manual reconstruction involves subjectively selecting and interpolating curves from points and the following creation of free-form NURBS object, the method developed excludes the human factor in the actual reconstruction thus allows for the fully automated manipulation of much larger amount of data.

\section{An algorithm for comparative analysis}

Parametric software was used not only for the phase of 3D reconstruction but also as a tool for analysis. Through an algorithmic definition, the accuracy of the three approaches for NURBS surface generation could be compared and evaluated. A random selection of 40000 points from the initial point cloud has been made, in order to be used in the evaluative algorithm. The points were projected (translated on the $\mathrm{Z}$ axis) on each of the reconstructed NURBS surfaces, deriving respectively from each of the initial meshes. The difference between the $Z$ value of the initial and the projected points (compared for each surface separately) are the parameter which indicates the accuracy of the rebuilt surface. As visible in fig. 6 , all values show an average deviation of around $2.6 \mathrm{~mm}$ from the original point cloud. The results also indicate that the surface reconstructed from the mesh, generated in MeshLab and rebuilt using contour lines is the most accurate one.

\section{Conclusions}

The paper presented an algorithmic-based approach to 3D reconstruction from point cloud aiming to automate the process of data elaboration and achieve accurate results with minimum effort. The methodology implements a reconstruction process where arbitrary modelling operations are avoided and replaced by algorithms able to compute automatically large amount of geometric data. Three different algorithmic approaches for rebuilding NURBS from mesh have been tested and their results compared with the initial point cloud to find out the precision level. The results indicated a high level of accuracy in all the implemented approaches, as the fastest surface was obtained with the algorithm projecting a grid of points on the mesh. The consistency in the level of precision among the three methods highlights an almost linear dependency with the input dataset, i.e. the more defined the point cloud, the more accurate the rebuilt surface. The methods can then be used in different cases, according to the analyzed building parts, without compromising on precision.

The intrinsic parametric nature of this approach offers the possibility of successive survey reiteration to improve progressively its precision, according the needs of the BIM model(s).

Next development of this research will consider larger building scale and the specifications of automatic procedure to obtain final output models with differential precision, according to the final use. The intention is to extend the workflow with the inclusion of the design and fabrication phase, to create customized building components for the adaptive reuse. 


\section{References}

[1] Arayici, Y., Towards Building Information Modelling for Existing Structures. Structural Survey, 26(3), pp. 210-222, 2008.

[2] Fai, S., Graham, K., Duckworth, T., Wood, N. \& Attar R., Building Information Modelling and Heritage Documentation. Proc. of the 23rd CIPA Symposium, Prague, 2011.

[3] Kensek, K. M., Building Information Modeling, Routledge, New York, 2014.

[4] Volk, R., Stengel, J. \& Schultmann F., Building Information Modeling (BIM) for Existing Buildings - Literature Review and Future Needs. Automation in Construction, 38, pp. 109-127, 2014.

[5] Oreni, D., Brumana, R., Banfi, F., Bertola, L., Barazzetti, L., Cuca, B., Previtali, M. \& Roncoroni, F., Beyond crude 3D Models: from Point Clouds to Historical Building Information Modeling via NURBS. Digital Heritage. Progress in Cultural Heritage: Documentation, Preservation, and Protection, eds. M. Ioannides, N. Magnenat-Thalmann, E. Fink, R. Žarnić, A.Y. Yen \& E. Quak, Springer International Publishing, Switzerland, pp. 166-175, 2014.

[6] Rinaudo, R., Chiabrando, F., Nex, F. \& Piatti, D., New Instruments and Technologies for Cultural Heritage Survey: Full Integration between Point Clouds and Digital Photogrammetry. Digital Heritage, eds. M. Ioannides, D. Fellner, A. Georgopoulos \& D.G. Hadjimitsis, Springer-Verlag: Berlin, pp. 56-70, 2010.

[7] Hichri, N., Stefani, C., De Luca, L. \& Veron, P., Review of the "As-Built BIM" Approaches. Int. Arch. Photogramm. Remote Sens. Spatial Inf. Sci., W1(XL-5), pp. 107-112, 2013.

[8] Remondino, F., Heritage Recording and 3D Modeling with Photogrammetry and 3D Scanning. Remote Sensing, 3(6), pp. 1104-1138, 2011.

[9] Berger, M., Tagliasacchi, A., Seversky, L.M., Alliez, P., Levine, J.A., Sharf, A. \& Silva, C.T., State of the Art in Surface Reconstruction from Point Clouds. Proc. of the 34th Eurographic Conf. eds. S. Lefebvre \& M. Spagnuolo, The Eurographics Association, pp. 161-185, 2014.

[10] Baumgart, B., Winged Edge Polyhedron Representation. Technical report, Stanford University for Advanced Research Projects Agency, Stanford, 1972.

[11] Lin, W.C. \& Chen, T.W., CSG-based Object Recognition Using Range Images. Proc. of the 9th Int. Conf. on Pattern Recognition. IEEE Comput Soc., pp. 99-101, 1988.

[12] Bernardini, F., Mittleman, J., Rushmeier, H., Silva, C. \& Taubin, G., The Ball-Pivoting Algorithm for Surface Reconstruction. IEEE Transactions on Visualization and Computer Graphics, 5(4), pp. 349-359, 1999.

[13] Dietrich, C.A., Scheidegger, C., Schreiner, J., Comba, J.L.D. \& Nedel, L.P., Edge Transformations for Improving Mesh Quality of Marching Cubes. IEEE Transactions on Visualization and Computer Graphics - TVCG, 15(1), pp. 150-159, 2009. 